\title{
The determination of fiber-bridging tractions in fiber-reinforced composites
}

\author{
Chao-Hsun Chen ${ }^{*}$, Ren-Roe Chang \\ Institute of Applied Mechanics, National Taiwan University, Taipei 10764, Taiwan, ROC
}

Received 16 October 1995; revised version received 9 April 1996

\begin{abstract}
A formulation is systematically derived for a fully or partially bridged straight crack in anisotropic materials such as fiber-reinforced composites. The dislocation density distribution corresponding to the measured COD of the bridged crack in composite specimens is obtained by the method of electronic-speckle-pattern interferometry (ESPI). With the dislocation density distribution as input in the equilibrium equations along crack surfaces, the fiber-bridging tractions exerted on the crack surfaces by intact bridging fibers can be determined. The tractions can be expressed either as a function of the position along the crack or a function of the crack opening displacement.
\end{abstract}

\section{Introduction}

Fiber-bridging of cracks, which may occur in composites made of strong ductile fibers and brittle matrix, play an important role in making the composites tougher. In general, the toughness of brittle solids such as ceramics and cement-like materials can be increased considerably by the use of fibers which bridge microcracks and provide additional resistance to fracturing (Marshall et al., 1985). Nemat-Nasser and Hori (1987) have studied the toughening mechanism of unidirectionally fiber-reinforced composites, which behave macroscopically like transversely isotropic materials. When the composites are stretched to such a degree that matrix cracks just occur and begin to propagate, the cracks will propagate without breaking the fibers. Therefore,

\footnotetext{
* Corresponding author.
}

when the matrix cracks open, the intact fibers crossing the crack may exert closing tractions, which are called fiber-bridging tractions, on the crack surfaces and thus reduce the crack-opening-displacement (COD) and the stress intensity factors at the crack tips. Fiber-bridging phenomena comprise debonding along the interfaces of matrix and bridging fibers and frictional sliding along the debonded part of the interfaces. It is quite difficult to predict the relation between fiber-bridging tractions and COD because the $\mathrm{COD}$, which can also be regarded as the length of fibers pulled out from matrix, depends on the depth of debonding and the sliding friction along the debonded part of the interface.

Many scholars have tried to analyze the relation between bridging tractions and COD by solving the problem of fiber pull-out from a single fiber composite unit cell. However, the boundary conditions specified by them are not exactly the same as those in real composite specimens. In order to determine fiber-bridging tractions in real composites and justify 
the relations between bridging tractions and COD proposed by other scholars, we propose a mathematical model to calculate bridging tractions from the measured crack opening profiles.

This paper is organized as follows: In Section 2 we recapitulate the important assumptions on which this study is based and some basic equations of Stroh formulation. In Sections 3 and 4 we follow Ting's (1988), by using new sum rules for Stroh formalism, to get the governing equations of fiber-bridged cracks formulated explicitly in real form in which the straight crack is in terms of the distribution of dislocation density and the bridging-force is modeled by continuously distributed tractions exerted on crack surfaces. In Section 5, we follow Nemat-Nasser and Hori (1987) by expressing dislocation density distribution as a series expansion of Chebychev polynomials to solve the governing equation and show how to calculate bridging tractions from measured COD profiles. In Section 6, a brief introduction of the experimental setup and specimen preparation are given. The experimental results and corresponding bridging tractions are demonstrated in Section 7.

\section{Stroh formulation}

Two important assumptions are as follows: the effective material properties of unidirectional-fiberreinforced composites are homogeneous transversely isotropic. The whole problem is approximated by a two-dimensional problem and analyzed by Stroh's formulation. Some important definitions and equations of Stroh's formulation are shown in the remainder of this section.

In a fixed rectangular coordinate system $x_{i}, i=1$, 2,3 , let $u_{i}$ and $\sigma_{i j}$ be the displacement and stress, respectively. Hooke's law and the equations of equilibrium are

$$
\begin{gathered}
\sigma_{i j}=C_{i j k s} u_{k, s} \\
C_{i j k s} u_{k, s j}=0
\end{gathered}
$$

in which $C_{i j k s}$ are the elastic constants, repeated indices imply summation, and a comma stands for differentiation. We assume that $C_{i j k s}$ are fully sym- metric and positive definite such that the strain energy is positive. Let

$$
\begin{gathered}
Q_{i k}(\theta)=C_{i j k s} n_{j}(\theta) n_{s}(\theta), \\
R_{i k}(\theta)=C_{i j k s} n_{j}(\theta) m_{s}(\theta), \\
T_{i k}(\theta)=C_{i j k s} m_{j}(\theta) m_{s}(\theta), \\
\mathbf{n}(\theta)=(\cos \theta, \sin \theta, 0), \\
\mathbf{m}(\theta)=(-\sin \theta, \cos \theta, 0)
\end{gathered}
$$

where $\theta$ is a real parameter and, in matrix notation,

$$
\begin{aligned}
\mathbf{N}_{1}(\theta) & =-\mathbf{T}^{-1}(\theta) \mathbf{R}^{\mathrm{T}}(\theta), \\
\mathbf{N}_{2}(\theta) & =\mathbf{T}^{-1}(\theta), \\
\mathbf{N}_{3}(\theta) & =\mathbf{R}(\theta) \mathbf{T}^{-1}(\theta) \mathbf{R}^{\mathrm{T}}(\theta)-\mathbf{Q}(\theta)
\end{aligned}
$$

The superscript $\mathrm{T}$ stands for the transpose. Define the incomplete integrals

$$
\begin{gathered}
\hat{\mathbf{S}}(\theta)=\frac{1}{\pi} \int_{0}^{\theta} \mathbf{N}_{1}\left(\theta^{\prime}\right) \mathrm{d} \theta^{\prime}, \\
\hat{\mathbf{H}}(\theta)=\frac{1}{\pi} \int_{0}^{\theta} \mathbf{N}_{2}\left(\theta^{\prime}\right) \mathrm{d} \theta^{\prime}, \\
\hat{\mathbf{L}}(\theta)=-\frac{1}{\pi} \int_{0}^{\theta} \mathbf{N}_{3}\left(\theta^{\prime}\right) \mathrm{d} \theta^{\prime}
\end{gathered}
$$

and the complete integrals

$\mathbf{S}=\hat{\mathbf{S}}(\pi), \quad \mathbf{H}=\hat{\mathbf{H}}(\pi), \quad \mathbf{L}=\hat{\mathbf{L}}(\pi)$

The three complete integrals $\mathbf{S}, \mathbf{H}, \mathbf{L}$ are the Barnett-Lothe tensors (Barnett and Lothe (1973)). The tensor $-L$ was first introduced by Stroh who denoted it by B (Stroh, 1958). The Barnett-Lothe tensors and the associated tensors $\hat{\mathbf{S}}(\theta), \hat{\mathbf{H}}(\theta), \hat{\mathbf{L}}(\theta)$ appear often in the solutions of two dimensional anisotropic elasticity problems.

\section{The basic real form solution for a line disloca- tion and force}

In this section we consider an infinite space which is subjected to a line dislocation and force at $r=0$. Ting (1988) has derived the displacement $u_{i}$ and the 
stress function $\varphi_{i}(i=1,2,3)$ which can be written as

$\mathbf{u}=-\frac{1}{\pi}(\ln r) \mathbf{h}-\mathbf{S}(\theta) \mathbf{h}+\mathbf{H}(\theta) \mathbf{g}+\mathbf{u}_{0}$

$\varphi=-\frac{1}{\pi}(\ln r) \mathbf{h}+\mathbf{S}(\theta) \mathbf{g}+\mathbf{L}(\theta) \mathbf{h}$

in which $\mathbf{g}$ and $\mathbf{h}$ are real constant vectors, $r=\left(x_{1}^{2}\right.$ $\left.+x_{2}^{2}\right)^{1 / 2}$, and $\mathbf{u}_{0}$ is the rigid body translation. The tractions on any radial plane, $t_{\theta}$, and on the cylindrical surface $r=$ constant, $t_{r}$, are

$\mathbf{t}_{\theta}=\frac{1}{\pi r} \mathbf{g}$

$\mathbf{t}_{r}=\frac{1}{\pi r}\left\{\mathbf{N}_{3}(\theta) h-\mathbf{N}_{1}^{\mathrm{T}}(\theta) \mathbf{g}\right\}$

It is noted that $\mathbf{t}_{\theta}$ is independent of $\theta$.

If $\mathbf{F}$ and $\mathbf{D}$ are the applied force and the line dislocation at $r=0$, respectively, from the equilibrium equation and the displacement discontinuity, we have

$\int_{0}^{2 \pi} \mathbf{t}_{r} r \mathrm{~d} \theta+\mathbf{F}=0$

$\mathbf{u}(0)-\mathbf{u}(2 \pi)=\mathbf{D}$

Substitutions of Eq. (3.1) into Eq. (3.6) and Eq. (3.2) into Eq. (3.5) lead to

$\mathbf{S}^{\mathrm{T}} \mathbf{g}+\mathbf{L h}=\mathbf{F}$

$\mathbf{H g}-\mathbf{S h}=\mathbf{D}$

Eqs. (3.7) and (3.8) can be solved for $g$ and $h$. If we multiply Eq. (3.7) by $\mathbf{S L}^{-1}$ and eliminate $\mathbf{S h}$ from Eq. (3.8) we have

$\mathbf{g}=\mathbf{L D}-\mathbf{S}^{\mathrm{T}} \mathbf{F}$

$\mathbf{h}=\mathbf{S D}-\mathbf{H F}$

Substitution of Eq. (3.9) into Eq. (3.3) leads to

$\mathbf{t}_{\theta}=\frac{1}{2 \pi r}\left(\mathbf{L D}-\mathbf{S}^{\mathbf{T}} \mathbf{F}\right)$

For a single dislocation, i.e. $\mathbf{F}=\mathbf{0}$, Eq. (3.11) can be reduced to

$\mathbf{t}_{\theta}=\frac{1}{2 \pi r} \mathbf{L D}$

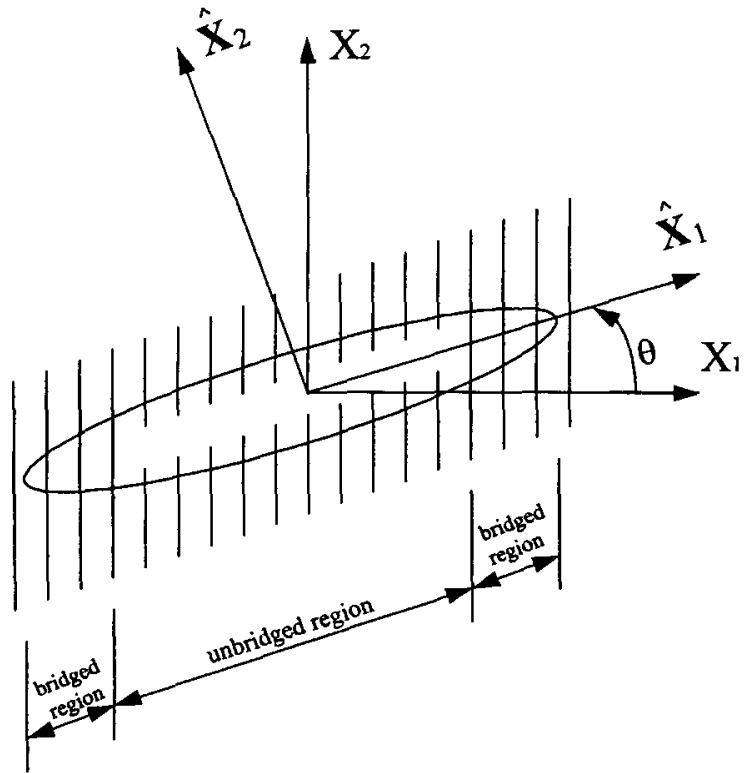

Fig. 1. A partially bridged straight crack in a unidirectionally fiber-reinforced composite: definition of coordinate systems.

Therefore the stress $\sigma_{2 i}$ along the $x_{1}$-axis induced by the dislocation can be written as

$\sigma_{2 i}=\frac{1}{2 \pi x_{1}} L_{i k} D_{k}$

For an oblique straight crack making an angle $\theta$ with the $x_{1}$-axis, we introduce the coordinates $\hat{x}_{1}$ and $\hat{x}_{2}$ as shown in Fig. 1 and observe that $b_{i}$ and $L_{i j}$ transform as first order and second order tensors,

$\hat{D}_{i}=R_{i p} D_{p}, \quad \hat{L}_{i j}(\theta)=R_{i p} R_{j q} L_{p q}(\theta)$

where $R_{i p}$ is a proper orthogonal tensor, rotating the $x_{p}$-coordinate system into the $\hat{x}_{i}$-coordinate system, and all indices in Eq. (3.14) take on values 1, 2. Define the dislocation density $\hat{b}_{i}$ as

$\hat{b}_{i}\left(\hat{x}_{1}\right)=-\frac{\mathrm{d} \hat{D}_{i}}{\mathrm{~d} \hat{x}_{1}}$

then a crack of length $2 c$ can be expressed in terms of the integration of the dislocation density $\hat{b}_{i}$ and the stresses $\sigma_{2 i}$ along the $\hat{x}_{1}$-axis due to the dislocations are

$\sigma_{2 i}\left(\hat{x}_{1}\right)=\frac{\hat{L}_{i k}}{2 \pi} \int_{-c}^{c} \frac{\hat{b}_{k}(\xi)}{\hat{x}_{1}-\xi} \mathrm{d} \xi$ 
where the symbol $\int$ represents the Cauchy principal value and $\hat{b}_{i}$ is the dislocation density.

\section{Formulation of partially bridged crack}

When the bridged area of the crack is much larger than the diameter and spacing of bridging fibers, the fiber-bridging force can be modeled by continuously distributed tractions exerted on the crack surfaces.

Fig. 2(a) shows schematically a bridged straight crack along the $\hat{x}_{1}$-axis which makes an angle $\pi / 2$ $-\theta$ with the direction of fibers. In Fig. 2(a) the farfield loading is denoted by $\hat{T}_{2 i}$ and the bridgingforce is denoted by $\hat{p}_{i}$. The problem shown in Fig. 2(a) is solved by superposing the two problems shown in Fig. 2(b) and (c) and the only condition to be satisfied is the boundary condition on crack surfaces, as shown below:

On the $\hat{x}_{1}$-axis from $(-c, 0)$ to $(c, 0)$, which corresponds to the location of the bridged crack, the stress components in Fig. 2(b) are

$\hat{\sigma}_{2 i}\left(\hat{x}_{1}\right)=\hat{T}_{2 i}$

The stress components in Fig. 2(c) are

$\hat{\sigma}_{2 i}\left(\hat{x}_{1}\right)=\frac{\hat{L}_{i k}}{2 \pi} \int_{-c}^{c} \frac{\mathrm{d} \xi}{\hat{x}_{1}-\xi} \hat{b}_{k}(\xi)$

then, the boundary condition at crack faces becomes

$$
\begin{gathered}
\hat{T}_{2 i}+\frac{L_{i k}}{2 \pi} \int_{-c}^{c} \frac{\mathrm{d} \xi}{\hat{x}_{1}-\xi} \hat{b}_{k}(\xi)=\hat{p}_{i}\left(\hat{x}_{1}\right), \\
-c<\hat{x}_{1}<c
\end{gathered}
$$

For an in-plane problem, Eq. (4.3) represents a pair of coupled singular integral equations which can be solved for the fiber-bridging tractions $\hat{p}_{i}$ whenever $\hat{b}_{i}$ and $\hat{T}_{2 i}$ are known. Before introducing our solution technique, we simplify the notation by setting

$\xi=c t, \quad \chi_{i}=\hat{x}_{i} / c, \quad \nu_{i}\left(\chi_{1}\right)=u_{i}\left(\hat{x}_{1}\right) / c$,

$b_{i}(t)=\frac{\hat{L}_{i i} \hat{b}_{i}\left(\hat{x}_{1}\right)}{2 \pi \sigma_{2 i}^{\infty}}$,

$$
\begin{aligned}
& t_{2 i}\left(\chi_{1}\right)=\frac{T_{2 i}\left(\hat{x}_{1}\right)}{\sigma_{2 i}^{\mathrm{x}}}, \\
& R_{i}\left(\chi_{1}\right)=\frac{K_{0} g\left(\hat{x}_{1}\right) c}{L_{i i} / 2 \pi}, \\
& Q_{1}=\frac{\hat{L}_{21}}{\hat{L}_{22}} \cot \theta, \quad Q_{2}=\frac{\hat{L}_{21}}{\hat{L}_{11}} \tan \theta
\end{aligned}
$$

Eq. (4.3) now becomes

$$
\begin{aligned}
& -\int_{-1}^{1} \frac{b_{i}(t)}{t-\chi_{1}} \mathrm{~d} t-Q_{i} \int_{-1}^{1} \frac{b_{j}(t)}{t-\chi_{1}} \mathrm{~d} t+t_{2 i} \\
& =p_{i}\left(\chi_{1}\right), \quad i, j=1,2, i \neq j
\end{aligned}
$$

In the following we have followed Nemat-Nasser and Hori (1987) by expressing dislocation density distribution as a series expansion of Chebychev polynomials to solve the governing equation and show how to calculate bridging tractions from measured COD profiles.
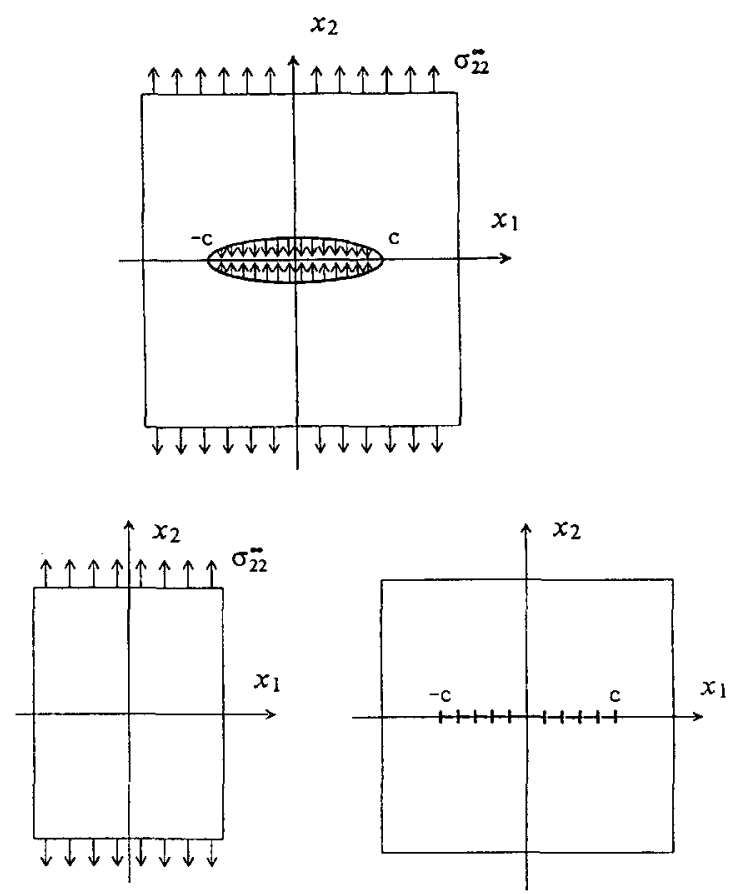

Fig. 2. Schematic bridged straight crack along the $\hat{x}_{1}$-axis which makes an angle $\pi / 2-\theta$ with the direction of fibers. 
It is noted that the function $b_{i}\left(\chi_{1}\right)$ has square-root singularities at $\chi_{1}= \pm 1$, the COD becomes

$$
\begin{gathered}
\nu_{i}\left(\chi_{1}\right)=W\left(\chi_{1}\right) \varphi_{i}\left(\chi_{1}\right), \\
W\left(\chi_{1}\right)=\sqrt{1-\chi_{1}^{2}}
\end{gathered}
$$

where $\varphi_{i}\left(\chi_{1}\right)$ is finite at $\chi_{1}= \pm$.

$W\left(\chi_{1}\right) \varphi_{i}\left(\chi_{1}\right)=0$ at $\chi_{1}= \pm 1$

Since the related orthogonal polynomials $\varphi_{i}$ are the Chebychev polynomials of the first kind, $T_{n}(t)$, from symmetry, it is seen that $b_{i}\left(\chi_{1}\right)=-b_{i}\left(-\chi_{1}\right)$. Then, the unknown function may be expressed as follows

$$
\begin{aligned}
& b_{1}(t)=\left(1-t^{2}\right)^{-1 / 2} \sum_{1}^{\infty} A_{1 n} T_{2 n-1}(t) \\
& b_{2}(t)=\left(1-t^{2}\right)^{-1 / 2} \sum_{1}^{\infty} A_{2 n} T_{2 n-1}(t)
\end{aligned}
$$

which must satisfy the following compatibility condition

$\int_{-1}^{1} b_{1}(t) \mathrm{d} t=0, \quad \int_{-1}^{1} b_{2}(t) \mathrm{d} t=0$

Substituting Eqs. (4.8a) and (4.8b) into Eq. (4.5) and using the following relations:

$$
\begin{aligned}
& \int_{-1}^{1} \frac{T_{n}(t)}{t-\chi}\left(1-t^{2}\right)^{-1 / 2} \mathrm{~d} t \\
& \quad= \begin{cases}\pi U_{n-1}(\chi), & |\chi|<1 \\
\pi G_{n}(\chi), & |\chi|>1\end{cases}
\end{aligned}
$$

where

$$
\begin{gathered}
U_{n}(\chi)=\csc \theta \sin [(n+1) \theta], \\
\theta=\cos ^{-1} \chi \\
G_{n}(\chi)=\frac{\left[\left(\chi^{2}-1\right)^{1 / 2}-\chi\right]^{n}}{(-1)^{n+1}\left(\chi^{2}-1\right)^{1 / 2}}
\end{gathered}
$$

we obtain the fiber bridging tractions as follows:

$$
\begin{aligned}
& -\sum_{n=1}^{\infty} \pi A_{i n} \frac{\sin [(2 n-1) \theta]}{\sin \theta} \\
& -Q_{i} \sum_{n=1}^{\infty} \pi A_{j n} \frac{\sin [(2 n-1) \theta]}{\sin \theta}+t_{2 i} \\
& =p_{i}\left(\chi_{1}\right), \quad i, j=1,2, i \neq j
\end{aligned}
$$

\section{The determination of fiber-bridging tractions from a measured crack opening profile}

To determine fiber bridging tractions from experiments, we have to accurately measure the COD at $M$ points, denoted by $t_{i}$ for $i=1$ through $M$, along the left half of the crack (because of symmetry, only measurement along the left half of the crack is enough). Then, with the dislocation density function expressed in Eqs. (4.8a) and (4.8b) and the following relations:

$$
\begin{aligned}
& \operatorname{COD}_{i}\left(\chi_{1}\right)=-\int_{-1}^{x_{1}} b_{i}(\xi) \mathrm{d} \xi \\
& \int_{-1}^{\chi_{1}} \frac{T_{n}(t)}{\left(1-t^{2}\right)^{1 / 2}} \mathrm{~d} t=-\frac{\sin (n \theta)}{n}
\end{aligned}
$$
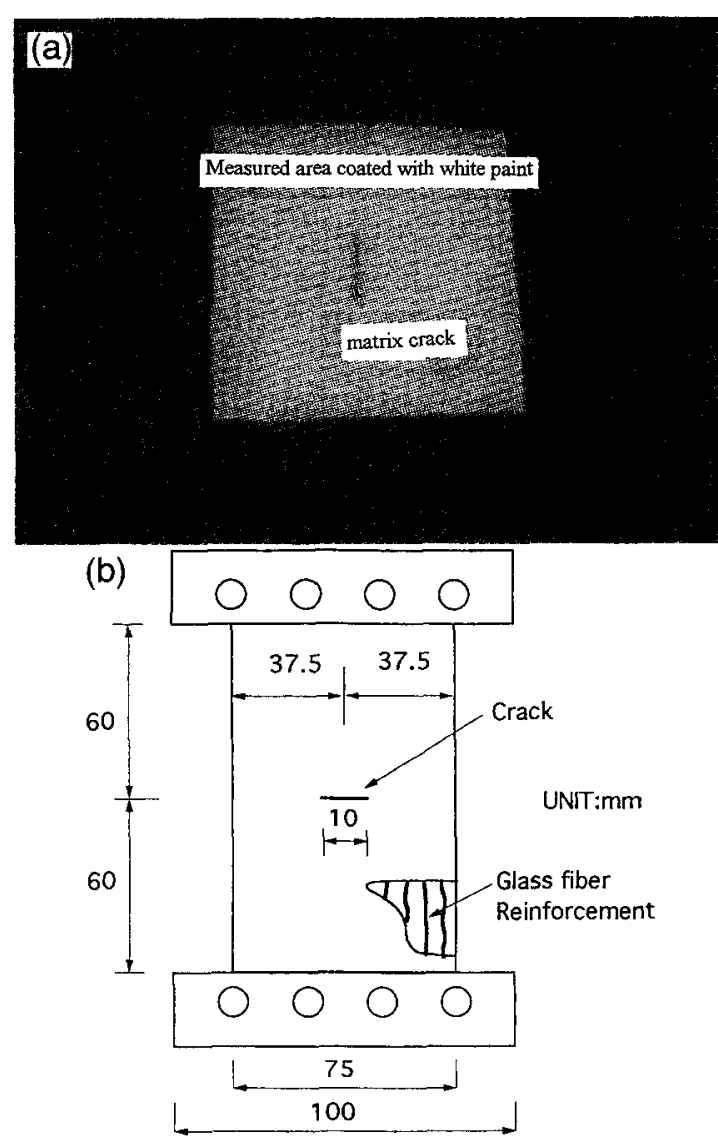

Fig. 3. Photograph of an unidirectional fiber-reinforced composite with a central crack. 
the coefficients $A_{1 n}$ and $A_{2 n}$ are solved from the following algebraic equations

$$
\begin{aligned}
& \sum_{n=1}^{N} A_{1 n} \frac{\sin \left[(2 n-1) \theta_{i}\right]}{2 n-1}=D_{1 i} \text { for } i=1 \text { to } N \\
& \sum_{n=1}^{N} A_{2 n} \frac{\sin \left[(2 n-1) \theta_{i}\right]}{2 n-1}=D_{2 i} \text { for } i=1 \text { to } N
\end{aligned}
$$

where $\theta_{i}=\cos ^{-1}\left(t_{i}\right)$, and $D_{1 i}, D_{2 i}$ are the COD in $\hat{x}_{1}$, and $\hat{x}_{2}$ directions measured at point $t_{i}$. If there is noise in the measured COD data, we can apply least square method to fitting a set of $A_{1 n}, A_{2 n}$ from data at $M$ points, where $M>N$. Then, we can find the corresponding bridging tractions by substituting the $A_{1 n}$ and $A_{2 n}$ into Eq. (4.13).

\section{Specimen preparation and experimental set up}

Epoxy (resin: Gy 260, hardner: B206, Epocone, Chemical Co., Taiwan) and glass fibers (Hexcel Knytex Weft D155) are made into our composite specimens by the RTM (resin transfer molding) method. Unidirectional glass fibers are fixed in a mold which has one inlet connecting to a resin tank and five outlets to a vacuum pump. Epoxy (resin and hardner mixed in weight ratio 5:1) is compressed into the mold after the vacuum pump is turned on. When the mold is filled, it is kept in an oven to make the material cured at $80^{\circ} \mathrm{C}$ for an hour. The specimen was cut into a $19 \mathrm{~cm} \times 8 \mathrm{~cm}$ rectangular plate.

The steps to make an artificial bridged crack are as follows: Since the melting points of epoxy and glass fiber are so much different, we wish to use the laserlight by choosing a suitable laser power to cut a $1 \mathrm{~cm}$ long central crack perpendicular to the fiber direction from both bottom and top sides, and still can leave the bridging fiber without damage. After the laser cut process, the specimen was investigated by microscopic observation. Results showed that only a very tiny portion of bridging fibers at both crack tips were damaged. This ensures us that the laserlight technique is capable to make the intact fiber bridging crack. For comparison, we also made a fiber-bridgingless (without fiber bridging) crack by choosing a

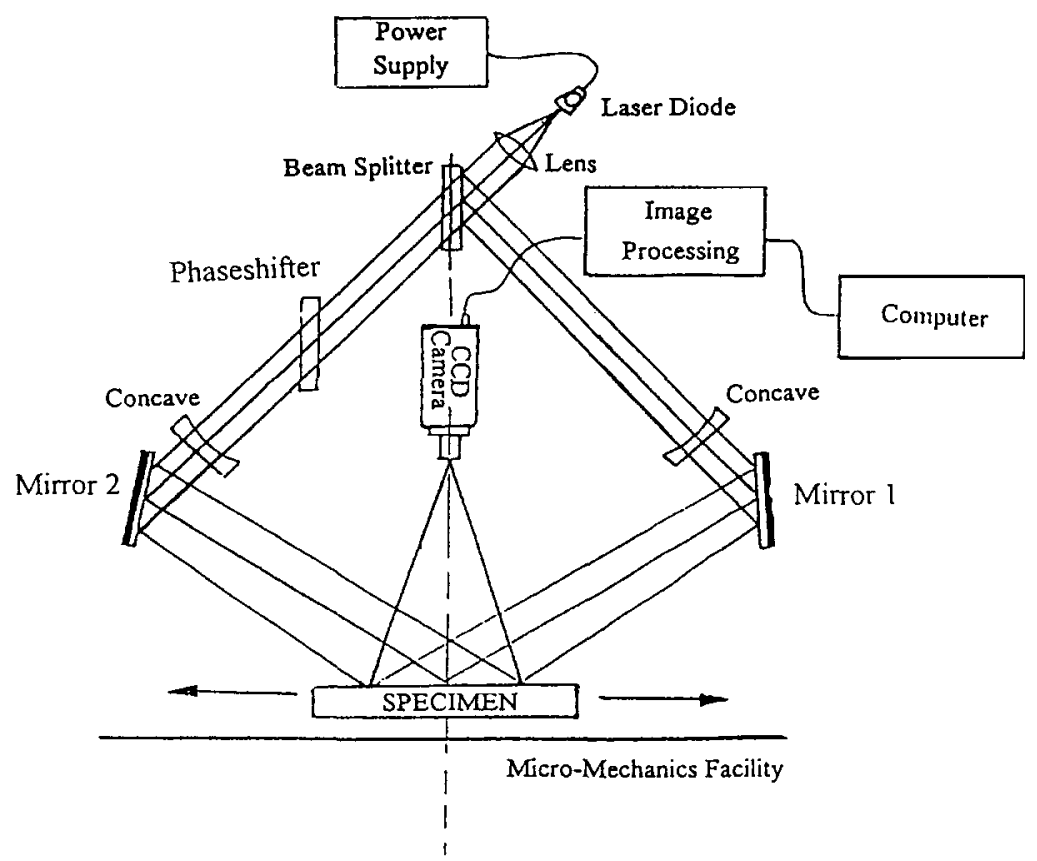

Fig. 4. The experimental setup for ESPI. 


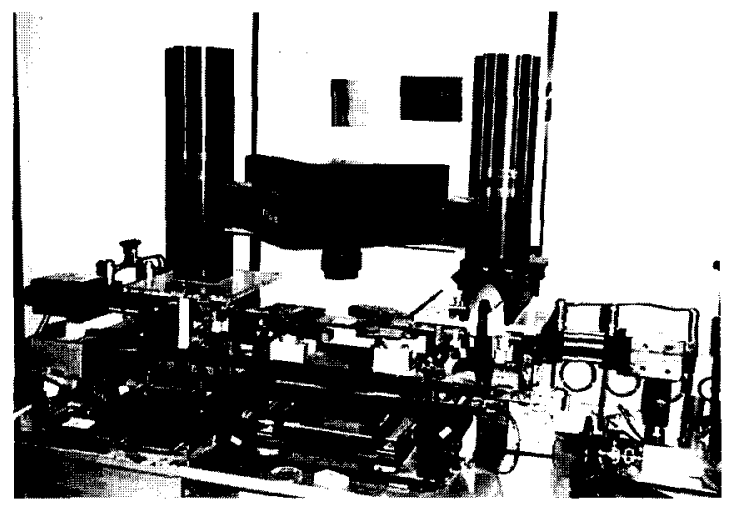

Fig. 5. Photograph of micro-loading testing machine.

laser power high enough to ensure that all the bridging fiber and matrix were fully broken. In order to enhance the reflective effect of the specimen surface
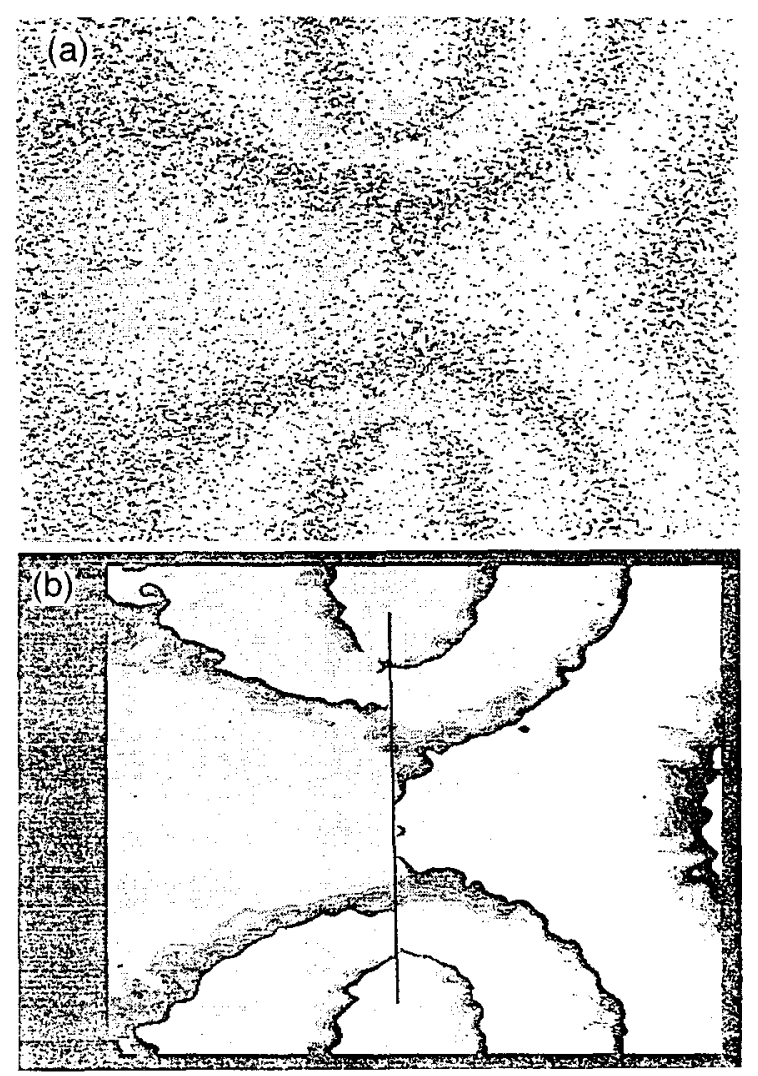

Fig. 6. (a) The fringe pattern of ESPI in the measured area as the unbridged crack opens under tensile force $5.2 \mathrm{~kg}$. (b) The result of post-image process of (a) (the straight line represents the position of the crack). (as shown in Fig. 3) to favor the optical measurement, $2 \times 2 \mathrm{~cm}^{2}$ around the crack was painted with white concrete paint. After the paint was dried out, a polished surface process was carried out. Thus the specimens with intact fiber-bridging and fiber-bridgingless central crack were ready for test.

A laser interferometry technique named ESPI (electronic speckle pattern interferometry) is used to measure the COD of the specimen. The experimental set up for ESPI is shown in Fig. 4. Laser beams are used to illuminate the surface of the sample. Due to the coherence of the light, the reflected light waves from each point of the surface interferes with each other and create a speckle pattern on the CCD array in the camera. All the information about the structure of the surface is in the speckle pattern. Whenever the
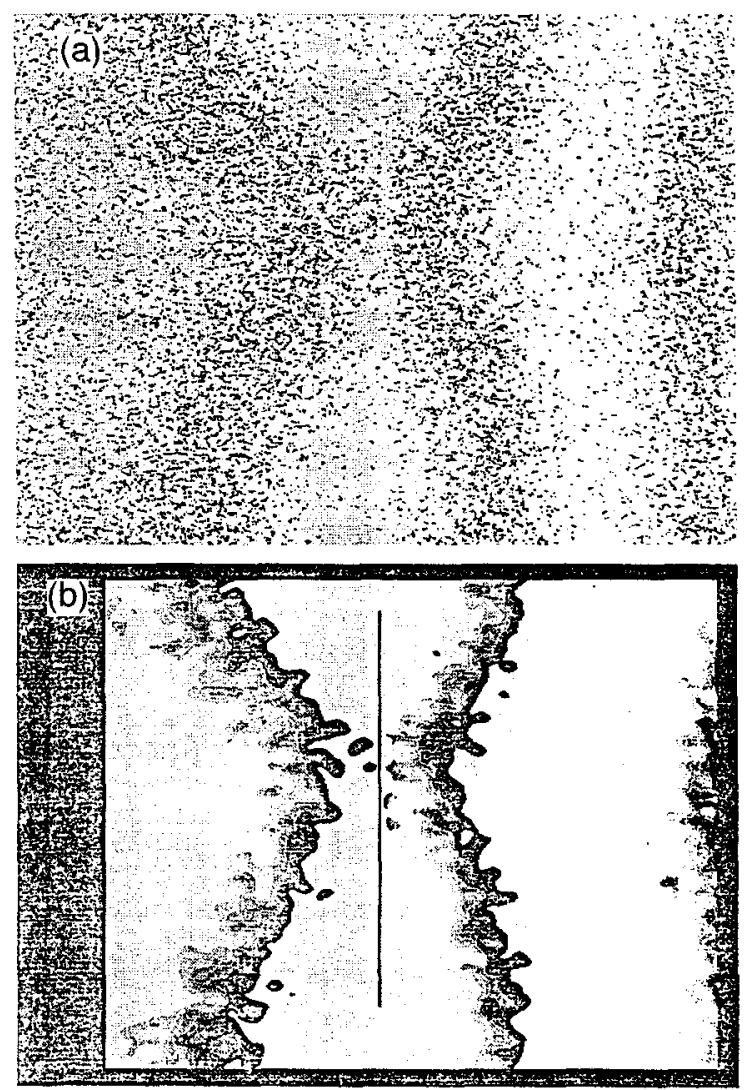

Fig. 7. (a) The ESPI fringe pattern of the fiber bridged matrix crack under far-field tensile force $7.5 \mathrm{~kg}$. (b) The result of post-image process of (a) (the straight line represents the position of the crack). 
structure of the surface changes, for example, by mechanical or thermal stress, the speckle pattern will change. Recording the speckle patterns of the specimen before and after it deforms by CCD camara, ESPI computer system can analyze the change in the speckle pattern and calculate the corresponding displacement field by a sophisticated image processing software. The facility SD800 ESPI in our laboratory uses a single-mode laser diode emitting coherent infrared light at $830 \mathrm{~nm}$ with power from 40 to 100 $\mathrm{mW}$. The system measures deformations in the order of the wavelength of the laser light from $50 \mathrm{~nm}$ to 20 $\mu \mathrm{m}$.

To avoid rigid body motion, which may exceed $20 \mu \mathrm{m}$, of the specimen during stretching, the specimen is stretched by a micromechanical test machine (shown as Fig. 5) which has two clamps that move simultaneously in opposite directions to keep the
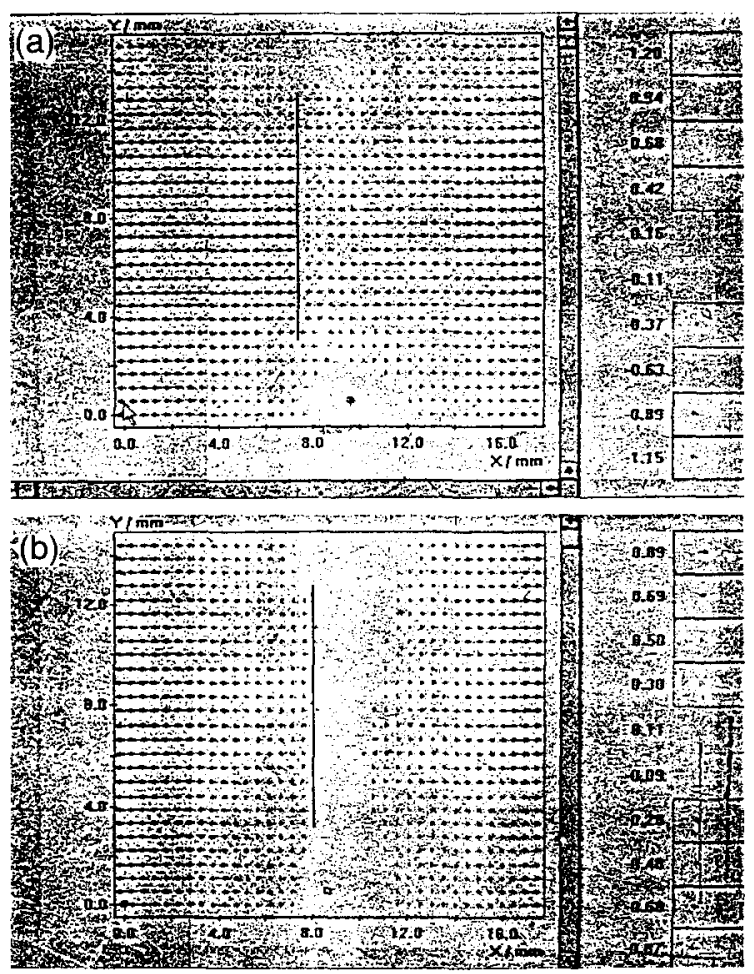

Fig. 8. (a) The displacement vector field of the inner measured area from computer-analysis diagram 6. (b) The displacement vector field of the inner measured area from computer-analysis diagram 7.

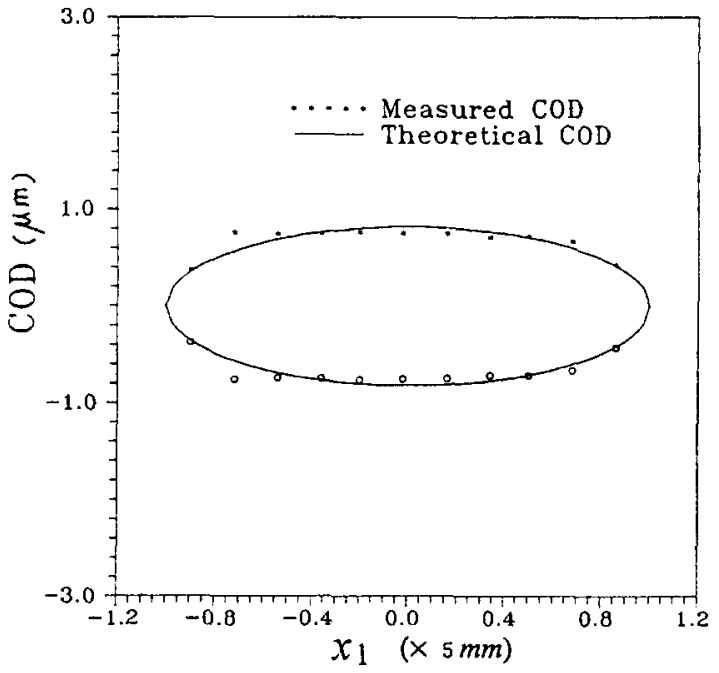

Fig. 9. The comparison between measured COD profile and the theoretical value of the unbridged crack.

center of the specimen, during stretching, from shifting. The stretch rate of this machine can be set in the range of $0.4 \mu \mathrm{m} / \mathrm{s}$ to $100 \mu \mathrm{m} / \mathrm{s}$ with a maximum loading of $100 \mathrm{~kg}$.

The effective properties $E_{1}, E_{2}, \nu_{21}$ and $G_{12}$, where subindex 2 stands for the direction parallel to fibers, are measured by tensile tests in $x_{1}, x_{2}$-axis and $45^{\circ}$ directions.

$$
\begin{gathered}
E_{1}=5594 \mathrm{MPa}, \quad E_{2}=24459 \mathrm{MPa}, \\
\nu_{21}=0.307, \quad G_{12}=2383 \mathrm{MPa}
\end{gathered}
$$

\section{Results and analysis}

Different object distances (the distance from the CCD camera of ESPI to the specimen, corresponding to different measured areas) or change tensile force (deciding the number of interference fringes on the screen) can be of influence to the computer analysis results of the interference fringes. In order to have the best ESPI measuring effect, such as size of measured area and magnitude of displacement, we have chosen a fiber-reinforced specimen with an unbridged central crack as our basis. After testing various experimental conditions, such as different 
object distances and different tensile loading forces, comparing the experimental results (the trend of displacement field and COD shown in the measured area) with the theoretical value, we have found that the most suitable experimental condition is as follows: The measured area was $29 \mathrm{~mm} \times 23 \mathrm{~mm}$ with tensile $11.7 \mathrm{~kg}$. Fig. $6(\mathrm{a}, \mathrm{b})$ show the fringe pattern of ESPI in the measured area as the unbridged crack opens under a tensile force of $5.2 \mathrm{~kg}$. Fig. 6(b)
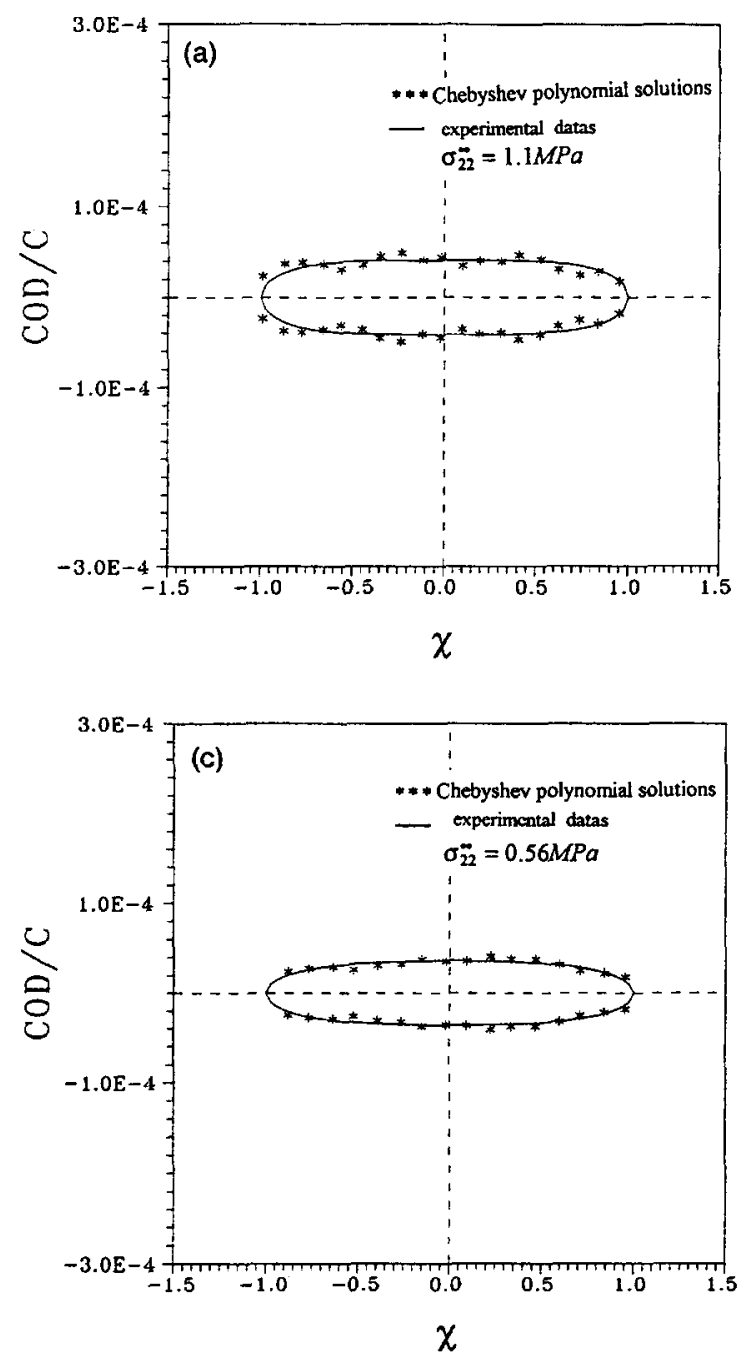

shows the result of a post-image process of Fig. 6(a), the straight line represents the position of the crack; while Fig. 7(a,b) show the ESPI fringe pattern of the fiber bridged matrix crack under a far-field tensile force of $7.5 \mathrm{~kg}$. Since the interference fringes correspond to the displacement field contours, the interference-fringe diagrams show that though the unbridged crack under smaller far-field tensile force, it still can have larger COD than the bridged crack.
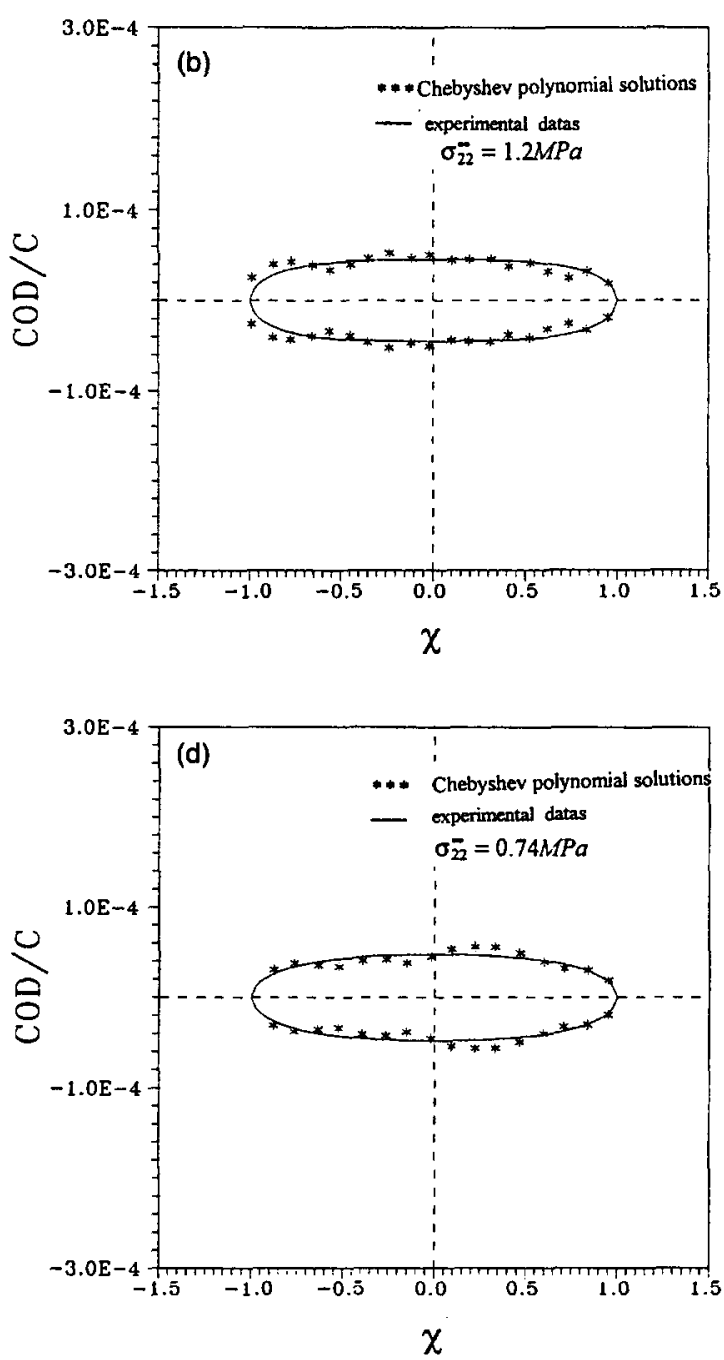

Fig. 10. The measured COD of the fiber bridged crack (the solid-line curves shown in the figures represent our Chebyshev polynomial solutions). 
Fig. 8(a,b) show the displacement vector field of the inner measured area from computer-analysis diagrams 6 and 7. We can apparently see the crack position from the vector field and obtain COD by calculating the difference between the displacement of upper and lower crack faces.

Fig. 9 plots the comparison between measured COD profile and the theoretical value of the unbridged crack. It shows that they both are in good agreements.
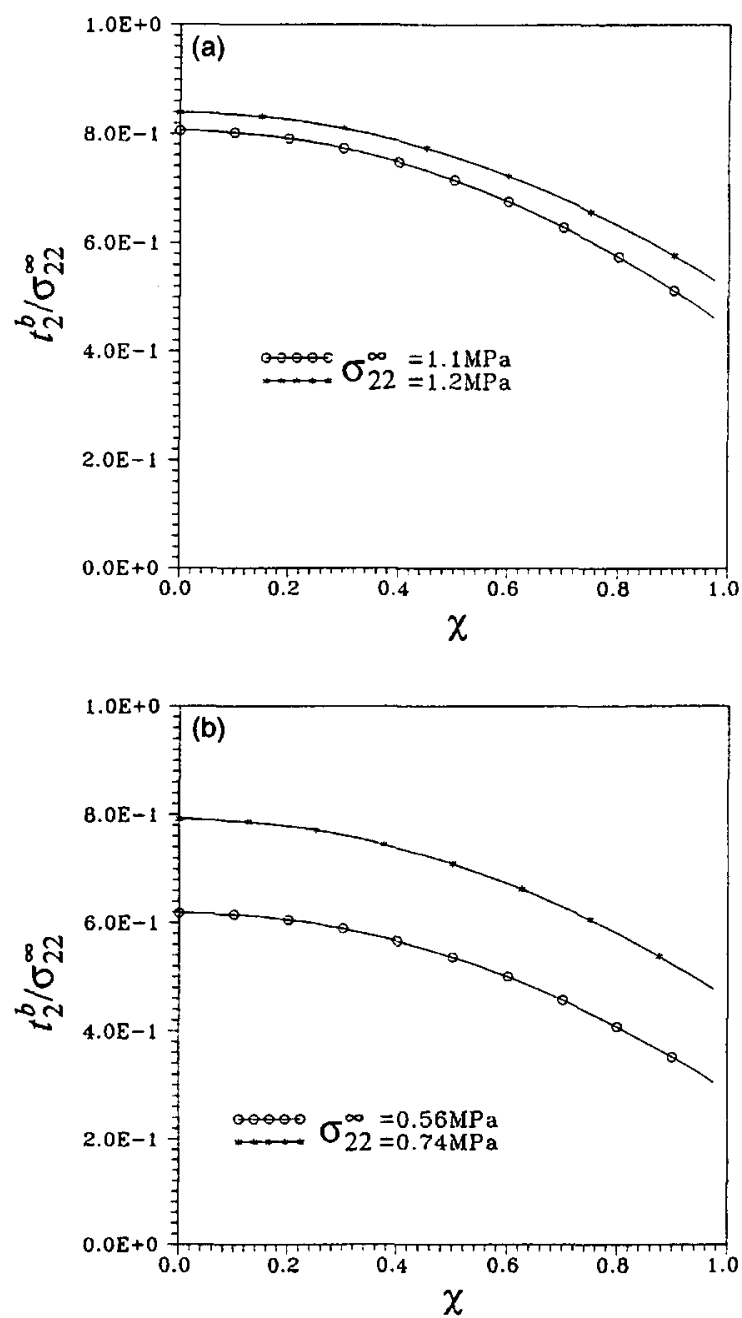

Fig. 11. (a) The bridging-traction distribution corresponding to the crack opening profile shown in Fig. $10(\mathrm{a}, \mathrm{b})$. (b) The bridgingtraction distribution corresponding to the crack opening profile shown in Fig. $10(\mathrm{c}, \mathrm{d})$.

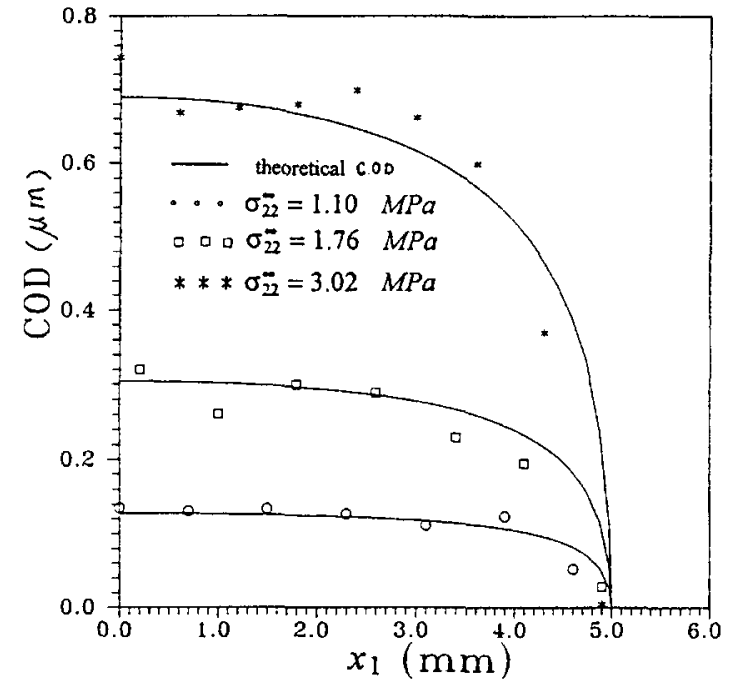

Fig. 12. COD under three different far-field loadings with interfacial properties $\mu=0.27, \sigma_{c}=0.18 \mathrm{MPa}, \sigma_{d}=3.0 \mathrm{MPa}$ substituted into the theory of Chen et al. (1995).

Fig. $10(\mathrm{a}-\mathrm{d})$ plot the measured COD of the fiber bridged crack, using the means derived in Section 5 to analyze the data. The results show that we only need two Chebyshev polynomial terms to approximate the crack opening profile (the solid-line curves shown in the figures represent our Chebyshev polynomial solutions).

Fig. 11(a, b) plot the bridging-traction distribution corresponding to the crack opening profile shown in Fig. 10(a, b) and (c, d), respectively. Since the COD decays to zero from middle to both tips of the crack, the bridging traction distribution falls off from the crack center to the tips.

Fig. 12 plots another COD result of the fiber bridged crack. We attempt using the bridging-traction-distribution analysis to handle the experimental result. The solid lines in Fig. 12 represent the COD under three different far-field loadings with interfacial properties $\mu=0.27, \sigma_{c}=0.18 \mathrm{MPa}, \sigma_{d}=3.0$ MPa substituted into the theory of Chen et al. (1995). We can see from the figure that in general the COD measured under three sets of different tensile loadings can be approximated by one set of material interfacial properties. This result can be seen as a justification to our theoretical model. 
From the above results we have demonstrated that the artificial fiber-bridged crack can be made by laser cutting process, and the distribution of bridging traction distribution can be calculated through the COD field measured by ESPI technique.

\section{Acknowledgements}

This work is supported by the National Science Council of ROC, NSC-81-0405-E002-01.

\section{References}

Barnett, D.M. and Lothe, J. (1973) Synthesis of the Sex tic and the Integral Formalism for Dislocation, Green's Function and
Surface Waves in An isotropic Elastic Solids. Phys. Norv. 7, 13-19.

Bamett, D.M. and Lothe, J. (1974) An Image Force Theorem for Dislocations in Anisotropic Bicrystals. J. Phys. F 1618-1635.

Chen, C.-H., Chang, R.-R. and Pao-Hwa J. (1995) On the FiberBridging of Cracks in Fiber-Reinforced Composites. Mech. Mater. 20, 165-181.

Marshall, D.B., N. Box and A.G. Evans (1985) The Mechanics of Matrix Cracking in Brittle-Matrix Fiber Composites. Acta Metall. 33(11), 2013-2021.

Nemat-Nasser S. and M. Hori (1987) Toughening by Partial or Full Bridging of Cracks in Ceramics and Fiber-Reinforced Composites. Mech. Mater. 6, 245-269.

Stroh, A.N. (1958) Dislocations and Cracks in Anisotropic Elasticity. Phil. Mag. 3, 625-646.

Ting, T.C.T. (1988) Line Forces and Dislocations in Anisotropic elastic Composite Wedges and Spaces. Phys. Stat. Sol. (b) 145, 81-90. 